\title{
On decimal and continued fraction expansions of a real number
}

by

\author{
C. FAivre (Marseille)
}

0. Introduction. Let $x$ be an irrational number. We deal with the problem of finding from the decimal expansion of $x$, the first $k$ (where $k$ is a given integer) partial quotients of the regular continued fraction expansion of $x$. More precisely, for each $n \geq 1$, denote by $x_{n}, y_{n}$ with $x_{n}<x<y_{n}$ the two consecutive $n$th decimal approximations of $x$. We assume that the integer $n$ is such that the numbers $x_{n}$ and $y_{n}$ have finite continued fraction expansions which coincide up to order $k$, i.e., $x_{n}=\left[\alpha_{0} ; \alpha_{1}, \ldots, \alpha_{k}, \ldots\right]$ and $y_{n}=\left[\alpha_{0} ; \alpha_{1}, \ldots, \alpha_{k}, \ldots\right]$ for some integers $\alpha_{i}$. Since the set of numbers which have a continued fraction which begins with $\alpha_{0}, \ldots, \alpha_{k}$ is an interval, it follows that $x=\left[\alpha_{0} ; \alpha_{1}, \ldots, \alpha_{k}, \ldots\right]$, in other words $\alpha_{0}, \alpha_{1}, \ldots, \alpha_{k}$ are precisely the first $k$ partial quotients of $x$. Writing the two rationals $x_{n}, y_{n}$ as a quotient $p / q$ of two integers, i.e., writing

$$
x_{n}=\frac{\left[10^{n} x\right]}{10^{n}} \text { and } y_{n}=x_{n}+\frac{1}{10^{n}},
$$

where $[y]$ denotes the largest integer $\leq y$ for each real number $y$, their continued fraction expansion may be computed exactly. In fact, for a rational number $p / q$, the continued fraction algorithm shows that we only have to perform operations on integers. This gives a practical method to compute the first $k$ partial quotients of an irrational number if we know as above the $n$ digits of its decimal expansion.

We can believe that for most irrational numbers $x$, the integer $n$ must be very large compared to $k$. Denote precisely by $k_{n}=k_{n}(x)$ the largest integer $k \geq 0$ such that we can write $x_{n}=\left[\alpha_{0} ; \alpha_{1}, \ldots, \alpha_{k}, \ldots\right]$ and $y_{n}=$ $\left[\alpha_{0} ; \alpha_{1}, \ldots, \alpha_{k}, \ldots\right]$ for some integers $\alpha_{i}$ with $\alpha_{0}=[x]$. Note that such a representation is always possible. In fact, $\left[x_{n}\right]=[x]=\alpha_{0}$ and $\left[y_{n}\right]=\alpha_{0}$ or

1991 Mathematics Subject Classification: Primary 11K50.

Key words and phrases: decimal expansion, transfer operators. 
$y_{n}=\alpha_{0}+1$ and in this last case we can write $y_{n}=\left[\alpha_{0} ; 1\right]$. Hence, $x_{n}, y_{n}$ will give $k_{n}$ partial quotients of $x$.

In [2] Lochs has proved the following beautiful and surprising result.

TheOREM (Lochs). For almost all irrationals $x$, with respect to Lebesgue measure, we have

$$
\lim _{n \rightarrow \infty} \frac{k_{n}(x)}{n}=\frac{6 \log 10 \log 2}{\pi^{2}} \simeq 0.9702 .
$$

Since the constant $0.9702 \ldots$ of the above theorem is rather close to 1 , one can almost say that for large $n$, the $n$ decimals determine the $n$ first partial quotients.

Consider two examples. For $x=\sqrt[3]{2}=1.259921 \ldots$, we have

$$
x_{5}=1.25992 \text { and } y_{5}=1.25993 .
$$

A computation shows that

$$
x_{5}=[1 ; 3,1,5,1,1,4,2,5,1,3] \text { and } y_{5}=[1 ; 3,1,5,1,1,5,5,1,2,1,4,3] .
$$

Therefore $k_{5}(x)=5$ and $x=[1 ; 3,1,5,1,1, \ldots]$. Thus we obtain from the five decimals of $x$ the first five partial quotients. As another example, the first 1000 decimals of $\pi$ give exactly 968 partial quotients (see [3]).

In this paper we improve the above theorem of Lochs.

Denote by $z_{0}$ the constant $(6 \log 10 \log 2) / \pi^{2}$. As probability measure on $[0,1]$ we will consider the Lebesgue measure denoted by $P$ in this paper. We prove the following theorem.

TheOREM 1 (main theorem). For all $\varepsilon>0$, the probability of the set of $x$ for which the distance of $k_{n}(x) / n$ to $z_{0}$ is greater than or equal to $\varepsilon$ decreases geometrically to 0 , i.e., there exist positive constants $C, \lambda$ (depending on $\varepsilon$ ) with $0<\lambda<1$ such that

$$
P\left(\left|\frac{k_{n}}{n}-z_{0}\right| \geq \varepsilon\right) \leq C \lambda^{n}
$$

for all integers $n \geq 1$.

The above theorem yields immediately that $\sum P\left(\left|k_{n} / n-z_{0}\right| \geq \varepsilon\right)<\infty$ for all $\varepsilon>0$. Then with the Borel-Cantelli lemma, we deduce easily as a corollary the theorem of Lochs.

The proof of the main theorem will show more precisely that

$$
\begin{aligned}
& \limsup _{n \rightarrow \infty} \frac{1}{n} \log P\left(\frac{k_{n}}{n} \leq z_{0}-\varepsilon\right) \leq \theta_{1}(\varepsilon) \quad\left(0<\varepsilon<z_{0}\right), \\
& \limsup _{n \rightarrow \infty} \frac{1}{n} \log P\left(\frac{k_{n}}{n} \geq z_{0}+\varepsilon\right) \leq \theta_{2}(\varepsilon),
\end{aligned}
$$


with

$$
\theta_{1}(\varepsilon)=\inf _{0<t<1 / 2} \frac{1}{t+1}\left(-t \log 10+\left(z_{0}-\varepsilon\right) \log \lambda(2-2 t)\right)<0
$$

and

$$
\theta_{2}(\varepsilon)=\inf _{\alpha>0}\left(\alpha \log 10+\left(z_{0}+\varepsilon\right) \log \lambda(2+2 \alpha)\right)<0 .
$$

In the above formulas $\lambda(2-2 t)$ and $\lambda(2+2 \alpha)$ are the dominant eigenvalues of some operators $L_{s}, s>1$ (transfer operators) defined in Section 2 .

The formulas giving $\theta_{1}$ and $\theta_{2}$ are interesting. If it is possible to extract further information about the location of the eigenvalues of the operators $L_{s}$ then we will have more precise estimates of $\theta_{1}$ and $\theta_{2}$.

We will also prove a result on approximation. For some irrationals $x$ it may happen that some decimals $x_{n}$ are better approximations of $x$ than $p_{n} / q_{n}$, i.e., $x-x_{n}<\left|x-p_{n} / q_{n}\right|$. We may take for example $x=\sqrt[3]{2}$ and $n=1,3,4,5$. However, the probability of this to happen decreases quickly to 0 as $n \rightarrow \infty$ according to the following theorem.

TheOREM 2. There exist positive constants $C, \mu$ with $0<\mu<1$ such that

$$
P\left(x-x_{n} \leq\left|x-\frac{p_{n}}{q_{n}}\right|\right) \leq C \mu^{n} \quad(n \geq 1) .
$$

The proof of the above theorem will show more precisely that

$$
\limsup _{n \rightarrow \infty} \frac{1}{n} \log P\left(x-x_{n} \leq\left|x-\frac{p_{n}}{q_{n}}\right|\right) \leq \theta
$$

with

$$
\theta=\inf _{\alpha>0} \frac{1}{\alpha+1}(\alpha \log 10+\log \lambda(2+2 \alpha))<0 .
$$

The following sections are devoted to the proof of Theorems 1 and 2 .

1. Conditional probabilities. If $\alpha_{1}, \ldots, \alpha_{i}$ are given integers $\geq 1$, the set of numbers in $[0,1]$ which have a continued fraction expansion which begins with $\alpha_{1}, \ldots, \alpha_{i}$ is an interval (a fundamental interval) denoted here as $I\left(\alpha_{1}, \ldots, \alpha_{i}\right)$. More precisely,

$$
I\left(\alpha_{1}, \ldots, \alpha_{i}\right)= \begin{cases}{\left[\frac{p_{i}}{q_{i}}, \frac{p_{i}+p_{i-1}}{q_{i}+q_{i-1}}\right]} & \text { if } i \text { is even, } \\ {\left[\frac{p_{i}+p_{i-1}}{q_{i}+q_{i-1}}, \frac{p_{i}}{q_{i}}\right]} & \text { if } i \text { is odd },\end{cases}
$$

where as usual

$$
\frac{p_{i}}{q_{i}}=\left[0 ; \alpha_{1}, \ldots, \alpha_{i}\right]
$$


In the following we will write $I\left(\alpha_{1}, \ldots, \alpha_{i}\right)=\left[b_{i}, c_{i}\right]$ for short. Let $r_{n i}=$ $\left[10^{n} b_{i}\right]$ and $r_{n i}^{\prime}=\left[10^{n} c_{i}\right]$, thus

$$
\frac{r_{n i}}{10^{n}} \leq b_{i}<\frac{r_{n i}+1}{10^{n}} \quad \text { and } \quad \frac{r_{n i}^{\prime}}{10^{n}} \leq c_{i}<\frac{r_{n i}^{\prime}+1}{10^{n}} .
$$

Let $x \in[0,1]$ be an irrational number. If $x \in\left[b_{i}, c_{i}\right]$, then $k_{n}(x) \geq i$ only when $x_{n}, y_{n}$ both belong to $\left[b_{i}, c_{i}\right]$. If $\left(r_{n i}+1\right) / 10^{n}>c_{i}$ then $y_{n}=\left(r_{n i}+\right.$ 1) $/ 10^{n}$, thus $y_{n} \notin\left[b_{i}, c_{i}\right]$. But if $\left(r_{n i}+1\right) / 10^{n} \leq c_{i}$, we will have $x_{n}, y_{n} \in$ $\left[b_{i}, c_{i}\right]$ only when $x \in\left[\left(r_{n i}+1\right) / 10^{n}, r_{n i}^{\prime} / 10^{n}\right]$ in the case $r_{n i} / 10^{n}<b_{i}$ and when $x \in\left[b_{i}, r_{n i}^{\prime} / 10^{n}\right]$ in the case $r_{n i} / 10^{n}=b_{i}$. Since

$$
c_{i}-b_{i}=\frac{1}{q_{i}\left(q_{i}+q_{i-1}\right)},
$$

we see that the conditional probability

$$
P\left(k_{n}<i \mid a_{1}=\alpha_{1}, \ldots, a_{i}=\alpha_{i}\right)
$$

is given by

$$
\begin{cases}1 & \text { if } \frac{r_{n i}+1}{10^{n}}>c_{i} \\ \left(\frac{r_{n i}+1}{10^{n}}-b_{i}+c_{i}-\frac{r_{n i}^{\prime}}{10^{n}}\right) q_{i}\left(q_{i}+q_{i-1}\right) & \text { if } \frac{r_{n i}+1}{10^{n}} \leq c_{i} \text { and } \frac{r_{n i}}{10^{n}}<b_{i} \\ \left(c_{i}-\frac{r_{n i}^{\prime}}{10^{n}}\right) q_{i}\left(q_{i}+q_{i-1}\right) & \text { if } \frac{r_{n i}+1}{10^{n}} \leq c_{i} \text { and } \frac{r_{n i}}{10^{n}}=b_{i} .\end{cases}
$$

For all $n \geq 1$, let $t_{n}$ and $v_{n}$ be the functions defined by

$$
t_{n}(y)=10^{n} y-\left[10^{n} y\right] \text { and } v_{n}(y)=1-t_{n}(y) .
$$

Since

$$
\frac{r_{n i}+1}{10^{n}}-b_{i}=\frac{v_{n}\left(b_{i}\right)}{10^{n}},
$$

we can write $P\left(k_{n}<i \mid a_{1}=\alpha_{1}, \ldots, a_{i}=\alpha_{i}\right)$ as

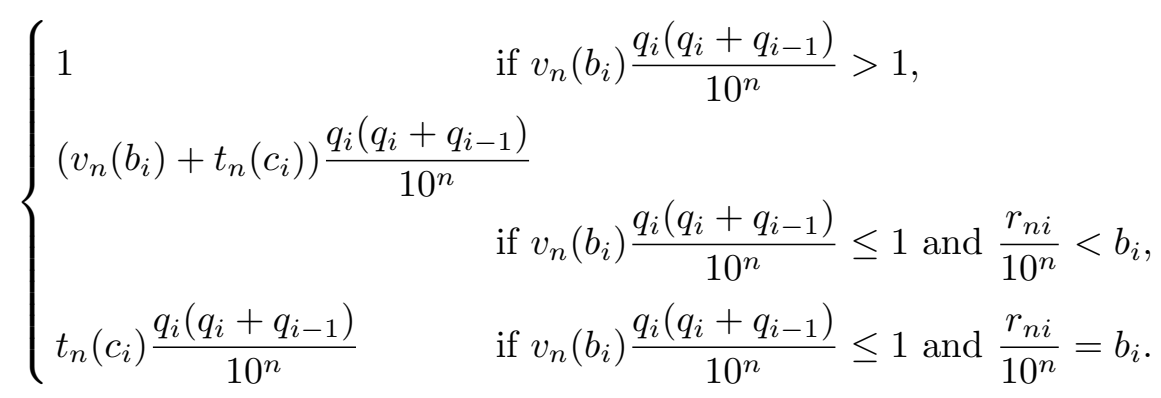


Note that $P\left(k_{n}<i \mid a_{1}=\alpha_{1}, \ldots, a_{i}=\alpha_{i}\right)$ is also equal to

$$
\begin{cases}1 & \text { if } \frac{q_{i}\left(q_{i}+q_{i-1}\right)}{10^{n}}>1, \\ \left(v_{n}\left(b_{i}\right)+t_{n}\left(c_{i}\right)\right) \frac{q_{i}\left(q_{i}+q_{i-1}\right)}{10^{n}} & \text { if } \frac{q_{i}\left(q_{i}+q_{i-1}\right)}{10^{n}} \leq 1 \text { and } \frac{r_{n i}}{10^{n}}<b_{i}, \\ t_{n}\left(c_{i}\right) \frac{q_{i}\left(q_{i}+q_{i-1}\right)}{10^{n}} & \text { if } \frac{q_{i}\left(q_{i}+q_{i-1}\right)}{10^{n}} \leq 1 \text { and } \frac{r_{n i}}{10^{n}}=b_{i} .\end{cases}
$$

In fact, if $v_{n}\left(b_{i}\right) q_{i}\left(q_{i}+q_{i-1}\right) / 10^{n} \leq 1$ and $q_{i}\left(q_{i}+q_{i-1}\right) / 10^{n}>1$, or equivalently if

$$
\frac{r_{n i}+1}{10^{n}} \leq c_{i} \quad \text { and } \quad c_{i}-b_{i}<\frac{1}{10^{n}}
$$

then we will necessarily have $r_{n i} / 10^{n}<b_{i}$ and $\left(r_{n i}+1\right) / 10^{n}=r_{n i}^{\prime} / 10^{n}$, thus

$$
\frac{v_{n}\left(b_{i}\right)+t_{n}\left(c_{i}\right)}{10^{n}}=c_{i}-b_{i}=\frac{1}{q_{i}\left(q_{i}+q_{i-1}\right)} .
$$

Let $T_{n i}$ be the random variable

$$
T_{n i}=P\left(k_{n}<i \mid a_{1}, \ldots, a_{i}\right),
$$

so, for the expectation of $T_{n i}$ we have

$$
E\left(T_{n i}\right)=P\left(k_{n}<i\right) .
$$

2. Transfer operators. Let $E=A_{\infty}(D)$ be the Banach space of bounded holomorphic functions on the disk $D=\{z:|z-1|<3 / 2\}$. The space $E$ is naturally endowed with the supremum norm $\|f\|_{\infty}=\sup _{z \in D}|f(z)|$. For each complex number $s$ with $\operatorname{Re}(s)>1$, we consider the following operator on $E$ :

$$
L_{s}(f)(z)=\sum_{n=1}^{\infty} \frac{1}{(n+z)^{s}} f\left(\frac{1}{n+z}\right) \quad(z \in D) .
$$

Note that for $s=2, L_{s}$ is the "analogue in $E$ " of the Perron-Frobenius operator of the Gauss transformation of continued fractions.

We recall in the following theorem some known properties of these operators $L_{s}$ (see for example [4] and [1]).

TheOREM 3. (a) $L_{s}$ is a nuclear operator of order 0 (hence it is compact in particular).

(b) For all real $s>1, L_{s}$ has a dominant eigenvalue $\lambda(s)>0$ of multiplicity 1.

(c) The map $s \rightarrow \lambda(s)$ is analytic.

(d) $\lambda(2)=1$ and $\lambda^{\prime}(2)=-\pi^{2} /(12 \log 2)$. 
A computation shows that the iterates of $L_{s}$ are given by the formula

$$
L_{s}^{n}(f)(z)=\sum_{k_{1}, \ldots, k_{n}} \frac{1}{\left(z q_{n-1}+q_{n}\right)^{s}} f\left(\frac{z p_{n-1}+p_{n}}{z q_{n-1}+q_{n}}\right),
$$

where $k_{1}, \ldots, k_{n}$ run over the integers $\geq 1$ and

$$
\frac{p_{n}}{q_{n}}=\left[0 ; k_{1}, \ldots, k_{n}\right] .
$$

In particular, we have

$$
L_{s}^{n}(f)(0)=\sum_{k_{1}, \ldots, k_{n}} \frac{1}{q_{n}^{s}} f\left(\frac{p_{n}}{q_{n}}\right) .
$$

Using the well-known formula

$$
\frac{q_{n-1}}{q_{n}}=\left[0 ; k_{n}, \ldots, k_{1}\right],
$$

we see by inverting the order of summation that we also have

$$
L_{s}^{n}(f)(0)=\sum_{k_{1}, \ldots, k_{n}} \frac{1}{q_{n}^{s}} f\left(\frac{q_{n-1}}{q_{n}}\right) .
$$

We use the operators $L_{s}$ to prove some probabilistic estimates about the denominators of the convergents $q_{n}$ which will be useful later. The letter $E$ denotes as usual the expectation operator.

Proposition 1. (i) For each $\alpha>0$, there exists a constant $C=C_{\alpha}$ such that

$$
E\left(\frac{1}{q_{n}^{2 \alpha}}\right) \leq C \lambda^{n}(2 \alpha+2) \quad(n \geq 1) .
$$

(ii) For each $t<1 / 2$, there exists a constant $C=C_{t}$ such that

$$
E\left(q_{n}^{2 t}\right) \leq C \lambda^{n}(2-2 t) \quad(n \geq 1) .
$$

Proof. (i) The expectation of $1 / q_{n}^{2 \alpha}$ is given by

$$
\begin{aligned}
E\left(\frac{1}{q_{n}^{2 \alpha}}\right) & =\sum_{k_{1}, \ldots, k_{n}} \frac{1}{q_{n}^{2 \alpha}} \cdot \frac{1}{q_{n}\left(q_{n}+q_{n-1}\right)} \\
& =\sum_{k_{1}, \ldots, k_{n}} \frac{1}{q_{n}^{2 \alpha+2}} \cdot \frac{1}{1+q_{n-1} / q_{n}},
\end{aligned}
$$

thus from (3), $E\left(q_{n}^{-2 \alpha}\right)=L_{2 \alpha+2}^{n}(f)(0)$, where $f(z)=1 /(1+z)$. From (b) of Theorem 3, we deduce that $\left|L_{2 \alpha+2}^{n}(f)(0)\right| \leq C \lambda^{n}(2 \alpha+2)$ for some constant $C>0$, thus (i) is proved.

(ii) Following the lines of (i), the expectation of $q_{n}^{2 t}$ is given by $E\left(q_{n}^{2 t}\right)=$ $L_{2-2 t}^{n}(f)(0)$ for $t<1 / 2$, with the same function $f$. This proves the result. 
3. Proof of the main theorem. First part. Since $0 \leq T_{n i} \leq 1$, we have for all $a>0$,

$$
E\left(T_{n i}\right) \leq a+P\left(T_{n i} \geq a\right)
$$

From (1), we have

$$
T_{n i} \leq\left(v_{n}\left(b_{i}\right)+t_{n}\left(c_{i}\right)\right) \frac{q_{i}\left(q_{i}+q_{i-1}\right)}{10^{n}},
$$

thus

$$
T_{n i} \leq \frac{4 q_{i}^{2}}{10^{n}} .
$$

Note that $E\left(q_{i}^{2}\right)=\infty$. Hence we cannot obtain a majorization of $E\left(T_{n i}\right)$ directly from the above inequality by taking expectations. However, we deduce

$$
P\left(T_{n i} \geq a\right) \leq P\left(\frac{q_{i}^{2}}{10^{n}} \geq \frac{a}{4}\right) .
$$

From the Markov inequality, for all $t>0$,

$$
P\left(\frac{q_{i}^{2}}{10^{n}} \geq \frac{a}{4}\right) \leq\left(\frac{4}{a}\right)^{t} 10^{-n t} E\left(q_{i}^{2 t}\right) .
$$

Hence from (4) and Proposition 1, where we restrict $0<t<1 / 2$, we get the inequality

$$
P\left(k_{n}<i\right)=E\left(T_{n i}\right) \leq a+\frac{C 4^{t} 10^{-n t} \lambda^{i}(2-2 t)}{a^{t}} .
$$

Taking $a=A^{1 /(t+1)}$ with $A=C 4^{t} 10^{-n t} \lambda^{i}(2-2 t)$, we obtain

$$
P\left(k_{n}<i\right) \leq 2 A^{1 /(t+1)} \text {. }
$$

Let $\left(i_{n}\right)$ be a sequence of integers $\geq 1$ such that

$$
\lim _{n \rightarrow \infty} \frac{i_{n}}{n}=z_{0}-\varepsilon
$$

and

$$
\frac{i_{n}}{n}>z_{0}-\varepsilon \quad \text { for all } n \geq 1 .
$$

From the last inequality for $P\left(k_{n}<i\right)$ we obtain for all $0<t<1 / 2$,

$$
\limsup _{n \rightarrow \infty} \frac{1}{n} \log P\left(k_{n}<i_{n}\right) \leq \frac{1}{t+1}\left(-t \log 10+\left(z_{0}-\varepsilon\right) \log \lambda(2-2 t)\right) .
$$

Thus

with

$$
\limsup _{n \rightarrow \infty} \frac{1}{n} \log P\left(\frac{k_{n}}{n} \leq z_{0}-\varepsilon\right) \leq \theta_{1}(\varepsilon)
$$

$$
\theta_{1}(\varepsilon)=\inf _{0<t<1 / 2} \frac{1}{t+1}\left(-t \log 10+\left(z_{0}-\varepsilon\right) \log \lambda(2-2 t)\right),
$$


since $P\left(k_{n} / n \leq z_{0}-\varepsilon\right) \leq P\left(k_{n}<i_{n}\right)$ from the choice of $\left(i_{n}\right)$. Now we show that $\theta_{1}(\varepsilon)<0$. In fact, consider for $u<1 / 2$ the function $h$ defined by

$$
h(u)=-u \log 10+\left(z_{0}-\varepsilon\right) \log \lambda(2-2 u) .
$$

By (d) of Theorem $3, h(0)=0$ and $h^{\prime}(0)=-\log 10+\left(z_{0}-\varepsilon\right) \pi^{2} /(6 \log 2)<0$. Thus if $t$ is sufficiently small, then $h(t)<0$, which implies that $\theta_{1}(\varepsilon)<0$ as asserted.

4. Proof of the main theorem. Second part. From (2) we have

$$
P\left(\frac{q_{i}\left(q_{i}+q_{i-1}\right)}{10^{n}}>1\right) \leq P\left(T_{n i}=1\right) \leq E\left(T_{n i}\right)=P\left(k_{n}<i\right),
$$

thus

$$
P\left(k_{n} \geq i\right) \leq P\left(\frac{10^{n}}{q_{i}\left(q_{i}+q_{i-1}\right)} \geq 1\right) .
$$

This last inequality can also be proved by noticing that if $k_{n} \geq i$ then $x_{n}, y_{n}$ are in the same $i$-fundamental interval as $x$, thus

$$
y_{n}-x_{n}=\frac{1}{10^{n}} \leq \frac{1}{q_{i}\left(q_{i}+q_{i-1}\right)},
$$

and this gives as above

$$
P\left(k_{n} \geq i\right) \leq P\left(\frac{10^{n}}{q_{i}\left(q_{i}+q_{i-1}\right)} \geq 1\right) .
$$

We can write

$$
P\left(k_{n} \geq i\right) \leq P\left(\frac{10^{n}}{q_{i}^{2}} \geq 1\right) .
$$

From the Markov inequality and Proposition 1, we get for all $\alpha>0$,

$$
P\left(k_{n} \geq i\right) \leq 10^{n \alpha} E\left(\frac{1}{q_{i}^{2 \alpha}}\right) \leq C 10^{n \alpha} \lambda^{i}(2+2 \alpha) .
$$

Now take a sequence $\left(i_{n}\right)$ of integers $\geq 1$ such that

$$
\lim _{n \rightarrow \infty} \frac{i_{n}}{n}=z_{0}+\varepsilon
$$

and

We have

$$
\frac{i_{n}}{n} \leq z_{0}+\varepsilon \quad \text { for all } n \geq 2 .
$$

$$
\limsup _{n \rightarrow \infty} \frac{1}{n} \log P\left(\frac{k_{n}}{n} \geq z_{0}+\varepsilon\right) \leq \theta_{2}(\varepsilon)
$$

with

$$
\theta_{2}(\varepsilon)=\inf _{\alpha>0}\left(\alpha \log 10+\left(z_{0}+\varepsilon\right) \log \lambda(2+2 \alpha)\right) .
$$


Now we prove that $\theta_{2}(\varepsilon)<0$. As in the first part of the proof, consider the function

$$
h(u)=u \log 10+\left(z_{0}+\varepsilon\right) \log \lambda(2+2 u) \quad(u>-1 / 2),
$$

and note that $h(0)=0$ and $h^{\prime}(0)<0$, thus $h(\alpha)<0$ for $\alpha$ sufficiently close to 0 and $\theta_{2}(\varepsilon)<0$.

5. Proof of Theorem 2. From $x-x_{n}=t_{n}(x) / 10^{n}$ and

$$
\left|x-\frac{p_{n}}{q_{n}}\right|<\frac{1}{q_{n}^{2}}
$$

we deduce

$$
P\left(x-x_{n} \leq\left|x-\frac{p_{n}}{q_{n}}\right|\right) \leq P\left(t_{n}<\frac{10^{n}}{q_{n}^{2}}\right) .
$$

For all $\varepsilon>0$ and $\alpha>0$, we can write

$$
P\left(t_{n}<\frac{10^{n}}{q_{n}^{2}}\right) \leq P\left(t_{n} \leq \varepsilon\right)+P\left(\frac{10^{n}}{q_{n}^{2}}>\varepsilon\right) \leq \varepsilon+\frac{C 10^{n \alpha} \lambda^{n}(2+2 \alpha)}{\varepsilon^{\alpha}} .
$$

The last inequality follows from the Markov inequality, Proposition 1, and the fact that for all $n \geq 1, t_{n}$ is distributed according to the uniform law on $[0,1]$. Taking

$$
\varepsilon=\left(C 10^{n \alpha} \lambda^{n}(2+2 \alpha)\right)^{1 /(\alpha+1)},
$$

we have

$$
P\left(t_{n}<\frac{10^{n}}{q_{n}^{2}}\right) \leq 2\left(C 10^{n \alpha} \lambda^{n}(2+2 \alpha)\right)^{1 /(\alpha+1)},
$$

thus

$$
\limsup _{n \rightarrow \infty} \frac{1}{n} \log P\left(t_{n}<\frac{10^{n}}{q_{n}^{2}}\right) \leq \theta
$$

with

$$
\theta=\inf _{\alpha>0} \frac{1}{\alpha+1}(\alpha \log 10+\log \lambda(2+2 \alpha))<0,
$$

which proves the theorem.

\section{References}

[1] C. Faivre, Distribution of Lévy constants for quadratic numbers, Acta Arith. 61 (1992), 13-34.

[2] G. Lochs, Vergleich der Genauigkeit von Dezimalbruch und Kettenbruch, Abh. Math. Sem. Univ. Hamburg 27 (1964), 142-144.

[3] -, Die ersten 968 Kettenbrüchen von $\pi$, Monatsh. Math. 67 (1963), 311-316. 
[4] D. Mayer, On the thermodynamic formalism for the Gauss map, Comm. Math. Phys. 130 (1990), 311-333.

Centre de Mathématiques et Informatique de l'Université de Provence

39 , rue Joliot Curie

13453 Marseille Cedex 13, France

E-mail: faivre@gyptis.univ-mrs.fr

Received on 26.4.1996

and in revised form on 7.4.1997 